\title{
A Short Note On The Electron-Positron Pair Creation
}

\section{Indicações Sobre a Criação de Pares Elétron-Pósitron}

\author{
Eduardo de Paiva ${ }^{1}$
}

\begin{abstract}
The electron-positron pair creation is one of the various mechanisms of interaction of photon radiation with matter and the pair can be produced when the photon interacts with an atomic nucleus or an electron. The photon energy thresholds $k_{t h}$ for these reactions are respectively $\sim 2 m_{0} c^{2}$ or $4 m_{0} c^{2}$ ( $m_{0}$ is the rest mass of one electron and $c$ is the speed of light in vacuum). An interesting feature of the electron-positron pair creation occurs when a very low energy photon $\left(k<<m_{0} c^{2}\right)$ collides with an ultra-relativistic electron $\left(E>>m_{0} c^{2}\right)$. In this note the kinematics of collision between a photon and an electron is reviewed and the particular and interesting feature of the triplet pair production when ultra-relativistic electrons interact with soft photons is briefly described.
\end{abstract}

Keywords: Pair production. Electron-positron pair. Triplet pair production.

\section{Resumo}

$\overline{\text { A criação de pares elétron-pósitron é um dos vários mecanismos de interação do fóton com a }}$ matéria e o par pode ser produzido quando o fóton interage com um núcleo atômico ou com um elétron. As energias limiares do fóton $k_{t h}$ para estas reações são respectivamente $\sim 2 m_{0} c^{2}$ ou $4 m_{0} c^{2}$ ( $m_{0}$ é a massa de repouso do elétron e $c$ é a velocidade da luz no vácuo). Um aspecto interessante da criação de par elétron-pósitron ocorre quando um fóton de energia muito baixa $\left(k<<m_{0} c^{2}\right)$ colide com um elétron ultra-relativístico $\left(E>>m_{0} c^{2}\right)$. Nesta nota a cinemática da colisão entre um elétron e um fóton é revista e o aspecto particular e interessante da produção de tripleto quando elétrons ultra-relativísticos colidem com fótons de baixíssimas energias é brevemente descrito.

Palavras-chave: Produção de pares. Par elétron-pósitron. Produção de tripleto.

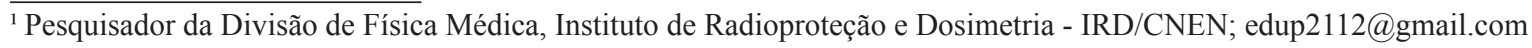




\section{Introduction}

The knowledge the of mechanisms of interaction between photon radiation and matter plays an important role in scientific and technological areas, such as radiation protection in industry and medicine, medical diagnosis and treatment of diseases, security surveillance in ports and airports, design of protective barriers against radiation in radiotherapy facilities, nuclear power plant shielding, and others applications. The interaction of photon radiation with matter is mainly governed by Rayleigh and Compton scattering, and the absorption processes of photoelectric effect, pair creation (in nuclear and electron field) and photodisintegration (KHAN; GIBBONS, 2014).

The different manners of how photon interacts with matter depend basically on its initial incident energy. At low photon incident energy $k(<10 \mathrm{keV})$ and high atomic number $Z$ of the absorbing medium the coherent or Rayleigh scattering, characterized by no energy transferring, i.e, the scattered and incident photons have the same energy, dominates the interaction process, and therefore in high-energy processes it can be neglected as for example in the medical practice of radiotherapy with the use of linear accelerators; the attenuation from the photoelectric effect is the principal mechanism of interaction at low incident photon energy due to the $1 / k^{3}$ energy dependence. Photoelectric effect is also proportional to the cube of the atomic number of absorber, and this dependence has a special importance in diagnostic radiology provided that the different $Z$ of the various tissues can increase or decrease the x-ray absorption; as the photon energy increases, the contribution of photoelectric effect drops sharply until the energy of photon is greater than the electron bind energy and Compton interaction begins to predominate. The Compton scattering falls off with energy of incident photon and is fairly independent of atomic number. The contribution of pair creation to the total attenuation process has a threshold energy of $2 m_{0} c^{2}\left(m_{0} c^{2}=0.511\right.$ $\mathrm{MeV}$, where $m_{0}$ is the rest mass of the electron and $c$ is the speed of light in vacuum) and increases with $Z^{2}$ and $k$. So, as the energy increases, the contribution due to the Compton scattering diminishes and the contribution of pair production gets larger. At higher energies of the incident photon radiation the pair production is the dominant process of interaction. Finally, at energies of the incident photon above $10 \mathrm{MeV}$ nuclear reactions, mainly with the emission of neutrons, begin to occur.

Now let us focus on the electron-positron pair formation, which is the dominant process at high photon energy as mentioned above. In reason of the energy and momentum conservation laws the photon materialization in an electron-positron pair is not possible in free space and has an energy threshold of $\sim 2 m_{0} c^{2}$ or $4 m_{0} c^{2}$ if the collision occurs respectively in the field of the nucleus (or proton) or in the field of the electron (in this case referred to as triplet pair formation, because the tracks of the recoil electron and the electron-positron pair created can be visualized in a cloud chamber forming a trident) (EISBERG; RESNICK, 1979; HEITLER, 1984). Electron-positron pair production by photons plays an important role in various physical phenomena (HAUG, 2004; MASTICHIADIS, 1991; MASTICHIADIS; PROTHEROE; SZABO, 1994), 
and both experimental and theoretical studies of these processes began soon after the prediction of existence of positron by Dirac as a consequence of the solutions of the relativistic wave equation for a free electron interacting with an electromagnetic field (DIRAC, 1928a, 1928b) and the further discovery of positron by Anderson when he studied the cosmic rays using a cloud chamber in which symmetrical tracks curving in opposite directions could be observed, evidencing a creation of an electron-positron pair (ANDERSON, 1932, 1933).

Electron-positron pairs can also be created when a photon collides with an ultra-relativistic electron $\left(E \gg m_{0} c^{2}\right)^{1}$ and in this case the minimum photon energy can be much lower than $m_{0} c^{2}$. In what follows the kinematics of collision between a photon and an electron is reviewed and the interesting feature of the triplet pair production when ultra-relativistic electrons interacts with soft photons will be briefly discussed.

\section{The Kinematics Of Interaction}

A schematic view of the collision between a photon and an electron is shown in Figure 1. Initially in the laboratory system of reference the incoming photon of energy $k$ and momentum $\boldsymbol{k}$ along the positive $x$-direction and the incoming primary electron of energy $E$ and momentum $\mathbf{P}_{\mathrm{e}}$ collide at an angle $\theta$, and in final state in the center-of-momentum system (also known as center-of-mass system, a system in which the total momentum is zero) the photon disappears and the three electrons (one recoil electron plus the pair created, the so called triplet pair production) move with individual energy and momentum respectively given by $E_{\mathbf{1}}^{\prime *}, E_{\mathbf{2}}^{\prime *}, E_{3}^{\prime *}$ and $\boldsymbol{P}_{\mathbf{1}}^{\prime *}, \boldsymbol{P}_{\mathbf{2}}^{\prime *}, \boldsymbol{P}_{\mathbf{3}}^{\prime *}$.

1 Sources of such ultra-high energy electrons can be found in astrophysical events and these electrons can also be produced in large particle linear accelerators.
The use of relativistic invariants (i.e., independent of a coordinate system in which it is calculated) to the initial and final state of a two-body collision process allow us to obtain information about the kinematics of the reaction without knowing further details of the interaction. The relation $E^{2}-P^{2} \mathrm{c}^{2}$ is an invariant (CHEW; LOW, 1959), where $E$ and $P$ denote the total energy and momentum of the system of particles. Applying it to the initial state in the laboratory system and to the final state in the center-of-mass system we can write

$$
\begin{aligned}
& (k+E)^{2}-\left(k+\boldsymbol{P}_{\theta}\right)^{2} c^{2}=\left(E_{1}^{\prime *}+E_{2}^{\prime *}+E_{3}^{\prime *}\right)^{2}- \\
& \left(\boldsymbol{P}_{1}^{\prime *}+\boldsymbol{P}_{2}^{\prime *}+\boldsymbol{P}_{3}^{\prime *}\right)^{2} c^{2}
\end{aligned}
$$

or

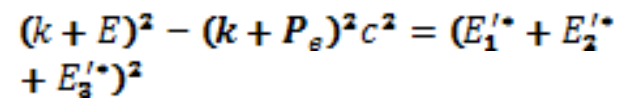

since $\boldsymbol{P}_{1}^{\prime *}+\boldsymbol{P}_{\mathbf{2}}^{\prime *}+\boldsymbol{P}_{3}^{\prime *}=\mathbf{0}$ by definition. Considering the horizontal axis and reminding that $|\boldsymbol{k}|$ $=k / c$ the above equation can be rewritten as

$$
2 k E+E^{2}-2 k P_{\theta} c \cos \theta-P_{\theta}^{2} c^{2}=\left(E_{T}^{\prime *}\right)^{2},
$$

where $E_{\mathbf{1}}^{\prime *}+E_{\mathbf{2}}^{\prime *}+E_{\mathbf{3}}^{\prime *}=E_{T}^{\prime *}$ is the total energy available in the center-of-mass system. Solving for $k$ we obtain

$$
k=\frac{E_{T}^{\prime * 2}-E^{2}+P_{\theta}^{2} c^{2}}{2 E-2 P_{\theta} c \cos \theta},
$$

or taking into account that $\frac{P_{\theta} c}{E}=\frac{v}{c}=\beta_{\theta}$, where $v$ is the velocity of the primary electron,

$$
E=\frac{E_{T}^{\prime * 2}-E^{2}\left(1-\beta_{\theta}^{2}\right)}{2 \mathrm{E}(1-\beta \operatorname{ecos} \theta)}
$$

The energy of the electron is $\frac{E=m_{0} c^{2}}{\sqrt{1-\beta_{\theta}^{2}}}$, or in another way $E^{2}\left(1-\beta_{\theta}^{2}\right)=m_{0}^{2} c^{4}$, and we can write 
Figure 1 - A simplified schematic view of the kinematics of collision between a photon and an electron.

\section{LABORATORY SYSTEM, before interaction}

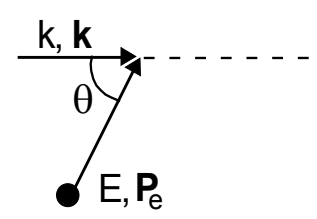

CENTER-OF-MASS SYSTEM, after interaction

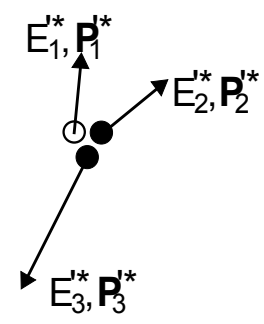

Source: author.

$$
k=\frac{E_{T}^{\prime * 2}-m_{0}^{2} c^{4}}{2 \mathrm{E}(1-\beta \operatorname{ecos} \theta)}
$$

Suppose now that the center-of-momentum system of reference moves horizontally with constant velocity $\beta_{c} c$ relative to the laboratory reference frame and the factor $\beta_{c}$ is defined by

$$
\beta_{c}=P \mathrm{c} / E \text {. }
$$

Again, $P$ and $E$ denote the total $x$-momentum and energy of the interacting particles. Thus, we can write

$$
\beta_{c}=\frac{\left(\frac{k}{c}+P_{\theta} \cos \theta\right) c}{k+E}=\frac{k+E \beta_{\theta} \cos \theta}{k+E} .
$$

\section{The Photon Energy Threshold}

Now, let us see what is the minimum photon energy necessary to create an electron-positron pair in a collision with a moving electron. At this point let us do the hypothesis that the triplet pair is formed at rest in the center-of-momentum frame, which corresponds to the minimum total energy $E_{T}^{\prime *}=\mathbf{3} m_{0} c^{\mathbf{2}}$ and from equation (6) we obtain

$$
k=\frac{\mathbf{4} m_{0}^{2} c^{4}}{E\left(1-\beta_{\theta} \cos \theta\right)} .
$$

Finally, for a given electron energy, it follows directly from the above expression that the minimum value of the photon energy occurs in a head-on collision, that is $\theta=\pi$,

$$
k_{t h}=\frac{4 m_{0}^{2} c^{4}}{E\left(1+\beta_{\theta}\right)} .
$$

And from (8) the corresponding factor $\beta_{c}$ is

$$
\beta_{c_{t h}}=\frac{k_{t h}-E \beta_{e}}{k_{t h}+E}
$$

For an ultra-relativistic electron $\beta_{e} \approx \mathbf{1}$ and for $k_{\text {th }}<E$ it can easily seen from the equation (11) that the center-of-mass frame moves to the left with respect to the laboratory frame.

The photon energy threshold (eq. 10) can also be obtained in an alternative way, considering the initial and final state in the laboratory frame. The conservation of energy and momentum gives

$$
\begin{gathered}
k+E=E_{\mathbf{i}}^{*}+E_{\mathbf{i}}^{*}+E_{\mathbf{3}}^{*} \\
\frac{k}{c}-P_{\theta}=-\left|P_{\mathbf{i}}^{*}+P_{\mathbf{2}}^{*}+P_{\mathbf{a}}^{*}\right|
\end{gathered}
$$

where $E_{\mathbf{1}}^{*}, E_{\mathbf{2}}^{*}, E_{\mathbf{3}}^{*}$ and $\boldsymbol{P}_{\mathbf{1}}^{*}, \boldsymbol{P}_{\mathbf{2}}^{*}, \boldsymbol{P}_{\mathbf{3}}^{*}$ are the final energy 
and momentum of each electron of the triplet pair. The assumption that the triplet pair is formed at rest in the center-of-momentum frame implies that in the laboratory frame the three particles move together to the left with constant velocity $\beta_{c_{t \boldsymbol{k}}} c$ and with equal energy and momentum $\left(E_{\mathbf{1}}^{*}=E_{\mathbf{2}}^{*}=E_{\mathbf{3}}^{*}\right.$ and $P_{1}^{*}=P_{2}^{*}=P_{3}^{*}$ ), so that

$$
\begin{gathered}
k+E=3 E_{1}^{*} \\
\frac{k}{c}-P_{\theta}=-3 P_{1}^{*} .
\end{gathered}
$$

Noting that $E_{1}^{*^{2}}=m_{0}^{2} c^{4}+P_{1}^{*^{2}} c^{2}$ we have

$$
k+E=\sqrt[3]{m_{0}^{2} c^{4}+P_{1}^{*^{2}} c^{2}}
$$

or

$$
k+E=3 \sqrt{m_{0}^{2} c^{4}+\frac{1}{9}\left(\frac{k}{c}-P_{\theta}\right)^{2} c^{2}} .
$$

Squaring this equation and solving for $k$ again we obtain the photon energy threshold:

$$
k_{t h}=\frac{4 m_{0}^{2} c^{4}}{E\left(1+\beta_{\theta}\right)} .
$$

Equations (18) and (11) reduce to the ordinary case commonly cited in physics textbooks, when the electron is at rest in the initial state in the laboratory system, i.e., $\beta_{e}=\mathbf{0}$ and $E=m_{0} c^{\mathbf{2}}$ which implies that $k_{\text {th }}=\mathbf{4} m_{0} c^{2}$ and $\beta_{c_{t \boldsymbol{k}}}=\mathbf{4} / \mathbf{5}$ (the center-of$\mathbf{4}^{\wedge}$ mentum moves to the right with constant velocity $\overline{\mathbf{5}}^{c}$ ).

In Figure 2 is shown how the photon energy threshold given by equation (10) varies as a function of the primary ultra-relativistic electron $\left(E \gg m_{0} c^{\mathbf{2}}\right.$ ). As can be seen from the Figure 2, the photon energy threshold can be as low as $\sim 10 \mathrm{eV}$ $\left(\sim 2 \times 10^{-5} m_{0} c^{\mathbf{2}}\right)$ for an electron energy about $\sim 50$ $\mathrm{GeV}\left(\sim 10^{\mathbf{5}} m_{0} c^{\mathbf{2}}\right)$.

At last it should be pointed out that when a very soft photon collides with an ultra-relativistic electron not only the triplet pair production can take place but also the inverse Compton scattering, when the energy of the scattered photon can be as high as the energy of primary electron (PAIVA, 2014). These processes can play an important role on the electron energy-loss and the triplet pair production can be a source of electronpositron pairs in electromagnetic pair cascade in astrophysical environments (MASTICHIADIS, 1991; MASTICHIADIS; PROTHEROE; SZABO, 1994).

\section{Final Remarks}

When a photon traverses the matter, it can interact in several ways. In particular, the electron-positron pair production plays an important role on these processes mainly at high photon energy. An interesting feature of the pair formation occurs when an ultra-relativistic electron collides with a very low energy photon and in final state the photon disappears giving rise to one pair electron-positron and one recoil electron, the triplet pair production. The photon energy threshold for the pair formation in a collision with an ultra-relativistic electron is briefly discussed and is shown that it can be as low as a few electrons-volt. 
Figure 2. The photon energy threshold as a function of the energy of primary electron.

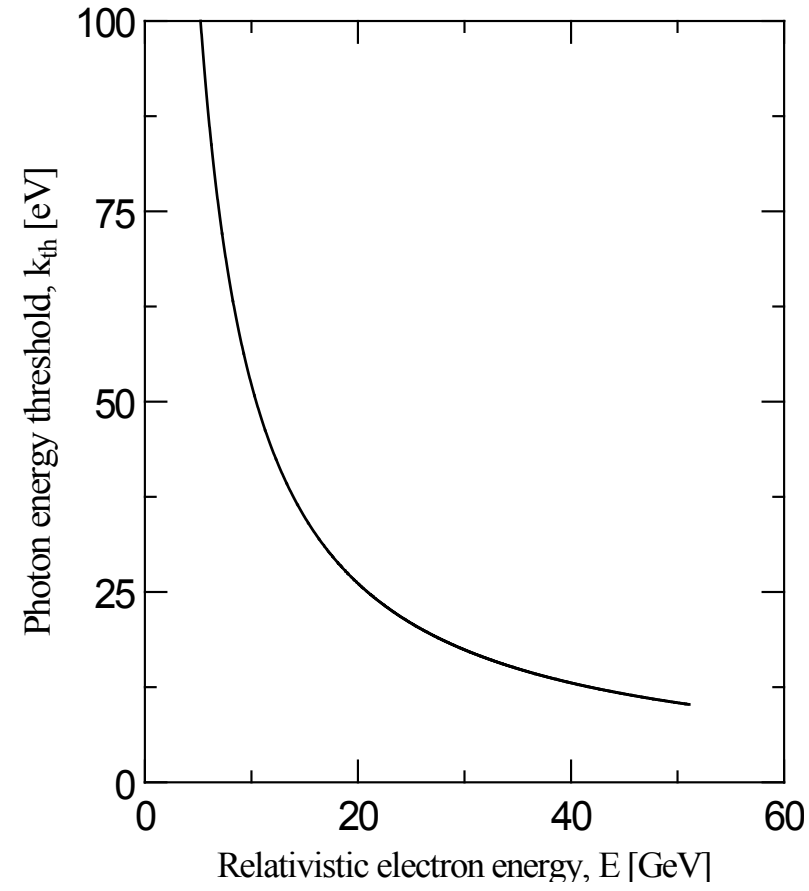

Source: author, from equation (10).

\section{REFERENCES}

ANDERSON, C. D. Energies of cosmic-ray particles. Physical Review, v. 41, p. 405-421, 1932.

. The positive electron. Physical Review, v. 43, p. 491-494, 1933.

CHEW, G. F.; LOW, F. E. Unstable Particles as Targets in Scattering Experiments. Physical Review, v. 113, p. 1640-1648, 1959.

DIRAC, P. A. M. The quantum theory of the electron. Proceedings of the Royal Society A, v. 117, p. 610-624, 1928a.

The quantum theory of the electron. Part II. Proceedings of the Royal Society A, v. 118, p. 351-361, $1928 \mathrm{~b}$.

EISBERG, R.; RESNICK, R. Física Quântica. Rio de Janeiro, Ed. Campus, 1979. Cap. 2, p. 69-74.

HAUG, E. Pair production by photons in a hot Maxwellian plasma. Astronomy and Astrophysics, v. 416, p. 437-440, 2004.
HEITLER, W. The quantum theory of radiation. New York, Dover Publications, 1984. Chap. 5, p. 256-268.

KHAN, F. M.; GIBBONS, J. P. The Physics of Radiation Therapy. Philadelphia, Williams \& Wilkins, 2014. Chap. 5, p. 62-70.

MASTICHIADIS, A. Relativistic electrons in photon fields: effects of triplet pair production on inverse Compton gamma-ray spectra. Monthly Notices of the Royal Astronomical Society, v. 253, p. 235-244, 1991.

MASTICHIADIS, A; PROTHEROE, R. J.; SZABO, A. P. The effect of triplet production on pair-Compton cascades in thermal radiation. Monthly Notices of the Royal Astronomical Society, v. 266, p. 910-916, 1994.

PAIVA, E. Sobre o espalhamento Compton inverso. Revista Brasileira de Ensino de Física, v. 36, p. 3303, 2014.
Recebido em 19 Março, 2017 - Received on March 19, 2017 Aceito em 12 Julho, 2017 - Accepted on July 12, 2017 